\title{
Periorbital Allergic Contact Dermatitis - The Most Common Aller- gens in the Czech Republic
}

\section{Miroslav Nečas* and Eliška Dastychová}

Faculty of Medicine, Dermatovenereological Clinic, St. Anne's Faculty Hospital Brno, Masaryk University Pekařská, Czech Republic

*Corresponding author: MUDr. Miroslav Nečas, Ph.D., Faculty of Medicine, Dermatovenereological Clinic, St. Anne's Faculty Hospital Brno, Masaryk University, Pekařská 53, 656-91 Brno, Czech Republic, Tel: +420543182809, Fax: +420543182794, E-mail: miroslav.necas@fnusa.cz

\begin{abstract}
Aims: 1) To determine actual frequency of sensitization to the most common contact allergens present in ophtalmic products. 2) To assess clinical relevance of the results and detect sources of sensitization.

Methods: A total number of 1881 patients (mean age 46.5 years, 357 men, 1,524 women) with periorbital dermatitis underwent patch tested between the years 2001 and 2016 and were retrospectively enrolled into our study.

Results: The highest frequency of sensitization was found with thimerosal $(9.7 \%)$, followed by neomycin sulfate $(2.6 \%)$, benzalkonium chloride $(1.3 \%)$, and parabens $(0.6 \%)$. In terms of clinical relevance however the most important allergen was neomycin sulfate.

Conclusion: The most important allergens in ophtalmologic products in our study were neomycin sulfate followed by benzalkonium chloride.

With every case of periorbital dermatitis patch tests should be considered, not only with commercial test allergens but with patient's actual ophtalmic preparations as well.
\end{abstract}

\section{Keywords}

Periorbital dermatitis, Patch tests, Contact allergens

\section{Introduction}

Periorbital dermatitis is quite a common problem in dermatology and also in ophthalmology. It may be either of allergic or non-allergic origin. Non allergic diseases include irritant contact dermatitis, atopic dermatitis (Figure 1), and seborrheic dermatitis. Periorbital allergic contact dermatitis may be caused by cosmetic products, nail polishes, hair dyes, certain airborne con-

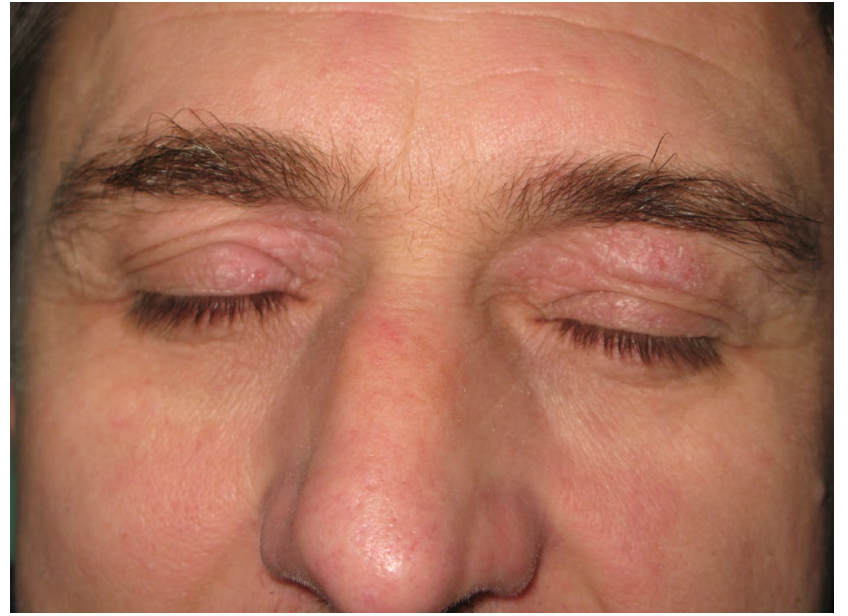

Figure 1: Atopic dermatitis in the periorbital region.

tact allergens (plants, epoxyde and other resins) or by ophtalmologic preparations [1]. The clinical appearance of allergic contact dermatitis in the periorbital region includes redness, often with eyelid swelling (Figure 2), in acute phase sometimes with oozing forming crusts, in subacute and chronic phase with less prominet redness and swelling but with more prominent scaling. The differentiation of allergic from non-allergic dermatitis as well as the identification of the cause of the allergy must be done by so called patch tests. The identifying of ophtalmologic drugs as the cause of periorbital dermatitis may be sometimes very difficult since patients frequently alternate usage of many different ophtalmologic products. Furthermore, there is no standardized ophthalmic patch test series and there are no pure com-

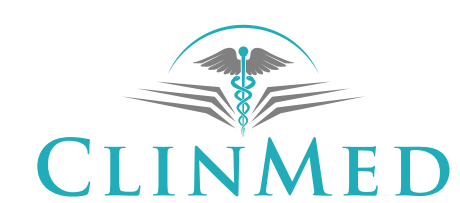

INTERNATIONAL LIBRARY
Citation: Nečas M, Dastychová E (2017) Periorbital Allergic Contact Dermatitis - The Most Common Allergens in the Czech Republic. Int J Ophthalmol Clin Res 4:074. doi.org/10.23937/2378-346X/1410074 Received: December 19, 2016: Accepted: July 28, 2017: Published: July 31, 2017

Copyright: (c) 2017 Nečas M, et al. This is an open-access article distributed under the terms of the Creative Commons Attribution License, which permits unrestricted use, distribution, and reproduction in any medium, provided the original author and source are credited. 


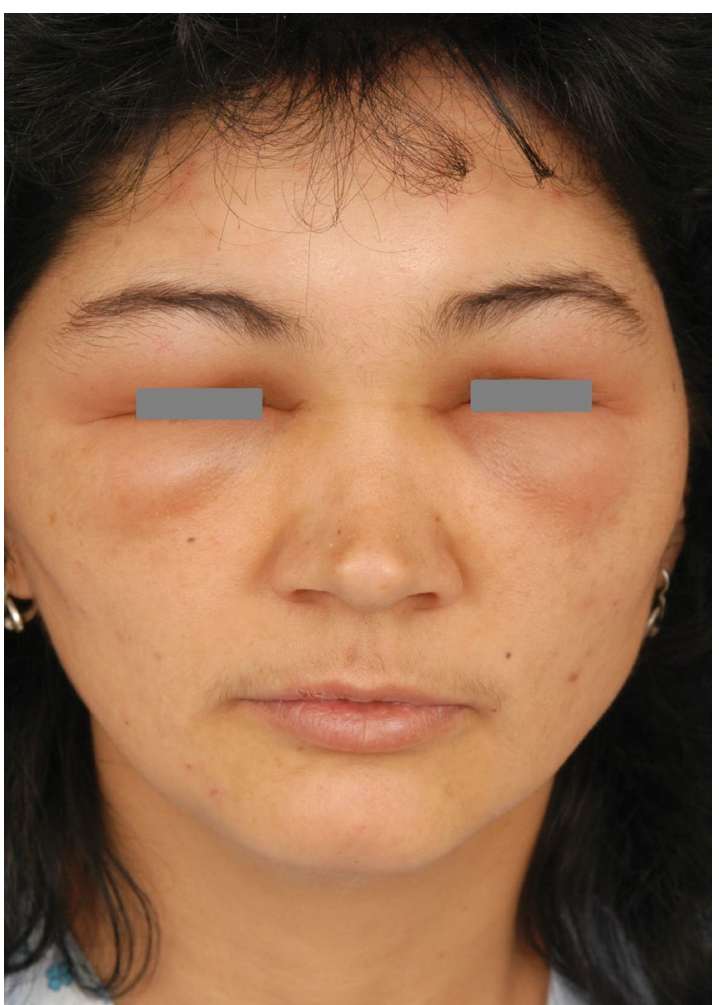

Figure 2: Acute periorbital allergic contact dermatitis with prominent swelling of the eyelids.

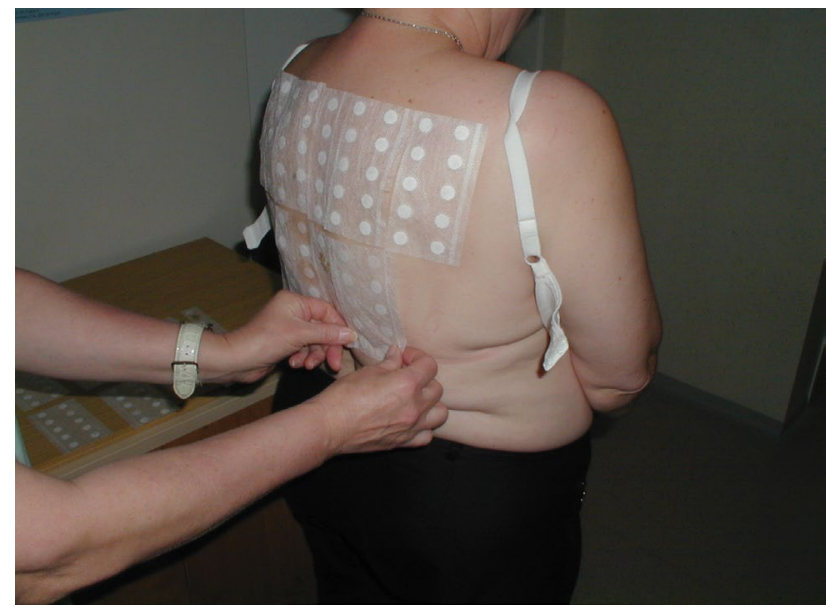

Figure 3: Application of patch tests.

pounds for patch testing [1]. Between the years 2001 and 2016 we have conducted a retrospective study among patients with periorbital dermatitis who were patch tested at the Dermatovenereology clinic of the St. Anne' s Faculty Hospital in Brno, Czech Republic, with the aim to determine actual frequency of sensitization to the most common contact allergens which are present in ophtalmic products.

\section{Material and Methods}

Between the years 2001 and 20161881 patients (mean age 46.5 years) with periorbital dermatitis were enrolled into our study. Of them were 357 men (mean age 43.9 years) and 1524 were women (mean age 47.0 years). All patients were patch tested, and clinical and anamnestic data were recorded before testing. A patch test (epicutaneous) is a diagnostic method used to identify the cause of allergic contact dermatitis (a delayed type of allergy). The principle of patch tests is to expose a small area of the skin (usually on the back) to the suspected allergen in a standardized amount, concentration, and vehicle. The patch may be a small piece of filter patch, plastic chamber, aluminum chamber or even a gel previously impregnated with an allergen (so called TRUE tests). The allergen is put on the patch and then placed on the patient's upper back skin, where patches are held in place with strips of adhesive tape, and remain undisturbed usually for 48 hours (Figure 3). Patches are then removed, and in the case of allergy an eczematous reaction (papules, papulovesicles) is visible in the tested area. Reaction severity is marked by + signs. Assessment is undertaken the next day ( 72 hours from application), the following day (after 96 hours), and in some cases (e.g. testing of corticosteroids) even one week after the application. This repeated reading is necessary, because in cases of true allergy the reaction increases over consecutive days, while in case of irritant (non-allergic) reaction, it usually decreases (the strongest reaction occurring on the day tests are removed, at which time the reaction begins to fade away). Patch tests methodology has been standardized by the International Contact Dermatitis Research Group (ICDRG) and we strictly follow these rules when patch testing our patients. With our patch tests, we have used Contact allergens of Chemotechnique Company and Lohmann \& Rauscher Curatest strips. Patch tests are contraindicated in cases of acute dermatitis, or when any rash is present in the test area, and when test area skin is heavily suntanned. Toxic or poisonous substances or substances with extremely high or low pH must not be patch tested. The tests are problematic in patients receiving simultaneous immunosuppressive treatment (may cause false negative results depending on the dose of immunosuppressants). The so called European baseline series (Table 1) of contact allergens was tested in all our patients along with additional suspected contact allergens depending on the patient's history. Retrospectively, frequency of sensitization to the most common contact allergens which are present in the ophtalmic products (thimerosal, benzalkonium chloride, neomycine, and paraben-mix) was assessed.

\section{Results}

Frequency of contact sensitization to selected allergens, which are present in ophtalmic products is shown in the Table 2. The highest frequency of sensitization was found with thimerosal ( $9.7 \%$ of patients), but among these patients just 1 case of allergy was caused by using an ophtalmic product with thimerosal. The second highest frequency was recorded with neomycine sulfate (2.6\%), where 22 sensitized patients had previously used an ophtalmic product containing neomycine sulphate. The third highest allergic sensitization was from benzalkonium chloride (1.3\%), and among pa- 
Table 1: European baseline series.

\section{Allergen, Concentration, Vehicle}

1. Potassium dichromate $0.5 \%$ pet.

2. Neomycin sulfate $20 \%$ pet.

3. Thiuram-mix $1 \%$ pet.

4. P-phenylenediamine $1 \%$ pet.

5. Kobalt chloride $1 \%$ pet.

6. Benzocaine $5 \%$ pet.

7. Formaldehyde $1 \%$ aq.

8. Colophony $20 \%$ pet.

9. Clioquinol $5 \%$ pet.

10. Balzam of Peru $25 \%$ pet.

11. N-Isopropyl-N-Phenyl-4-Phenylenediamine (IPPD) $0.1 \%$ pet.

12. Lanolin alcohol $30 \%$ pet.

13. Mercapto-mix $2 \%$ pet.

14. Epoxy resin $1 \%$ pet.

15. Paraben-mix $16 \%$ pet.

16. 4-tert-butylphenolformaldehyde resin $1 \%$ pet.

17. Fragrance-mix $8 \%$ pet.

18. Quaternium-15 1\% pet.

19. Nickel sulfate $5 \%$ pet.

20. Methylisothiazolinone + methylchloroisithiazolinone (Kathon CG) $0.01 \%$ aq.

21. 2-Merkaptobenzothiazole $2 \%$ pet.

22. Sesquiterpene lactone-mix $0.1 \%$ pet.

23. Prim in $0.01 \%$ pet.

24. Budesonide $0.01 \%$ pet.

25. Tixocortol-21-pivalate $0.1 \%$ pet.

26. Methyldibromo glutaronitrile $0.5 \%$ pet.

27. Lyral 5\% pet.

28. Fragrance-mix II $14 \%$ pet.

29. Methylisothiazolinone $0.2 \%$ aq.

30. Textile dye-mix $6.6 \%$ pet.

Table 2: Frequency of sensitization to the selected allergens used in ophtalmologic products.

\begin{tabular}{|l|l|l|l|l|l|l|}
\hline Allergen & N & $\%$ & R/NR & \multicolumn{3}{l|}{ Source } \\
\hline Thimerosal & 182 & 9.7 & $1 / 182$ & 0 & 1 & 181 \\
\hline Neomycin & 48 & 2.6 & $20 / 48$ & 0 & 20 & 28 \\
\hline $\begin{array}{l}\text { Benzalkonium } \\
\text { chloride }\end{array}$ & 25 & 1.3 & $3 / 25$ & 15 & 3 & 7 \\
\hline Parabens & 11 & 0.6 & $0 / 11$ & 8 & 0 & 3 \\
\hline
\end{tabular}

Legend: $\mathrm{N}=$ Number of Positive Reactions to a Given Allergen; $\%=$ Frequency of Contact Sensitization in \%; R = Relevant Reaction; NR = Non-Relevant Reaction; Cosm. = Cosmetic Product; Ppht. $=$ Ophtalmologic Product.

tients allergic to benzalkonium chloride, 3 patients had been previously using ophtalmic products containing the allergen (Figure 4). The least common was sensitization to parabens $(0.6 \%)$, where none of the patients had used any ophtalmic product containing parabens (Figure 5 and Figure 6).

\section{Discussion}

Thimerosal (Merthiolate or Thiomersalate) or sodium ethylmercury salicylate is an organic mercury compound which is used as a preservative and disinfectant in contact lenses, and eye solutions, vaccines, and tattoo inks. Thimerosal is composed of two radicals: an organic

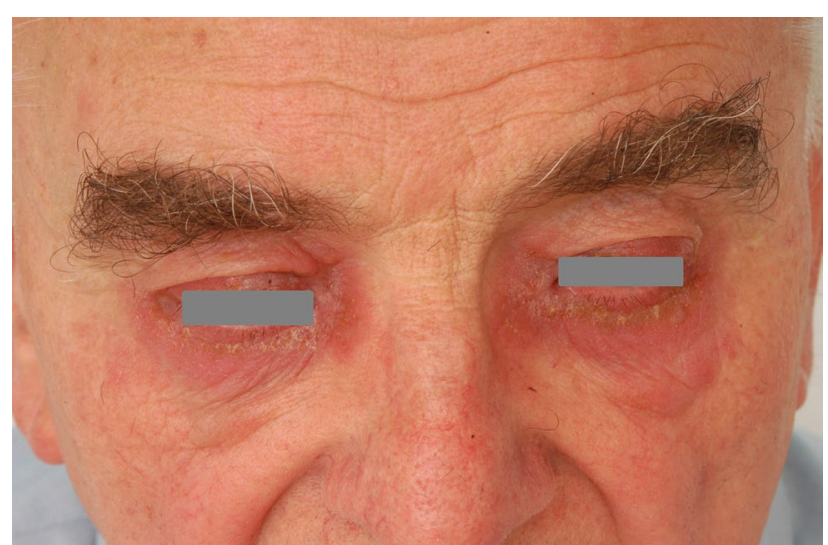

Figure 4: Allergic contact dermatitis to benzalkonium chloride.

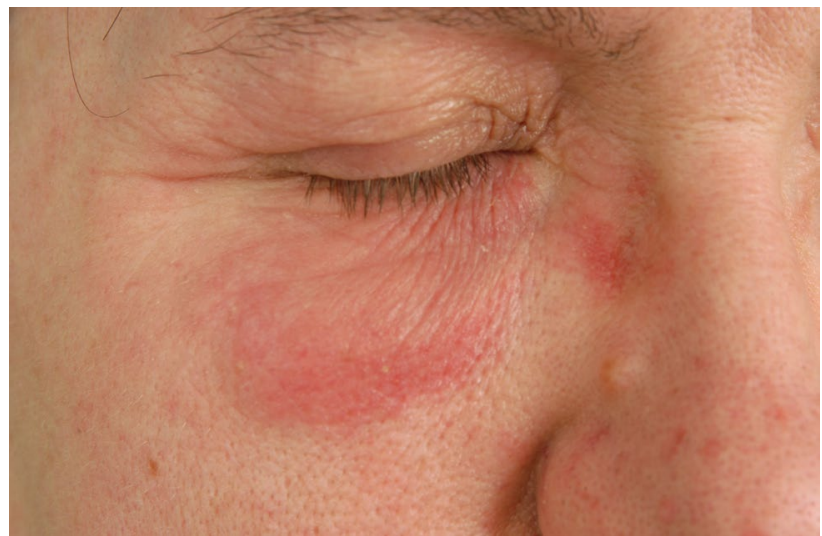

Figure 5: Allergic contact dermatitis to parabens.

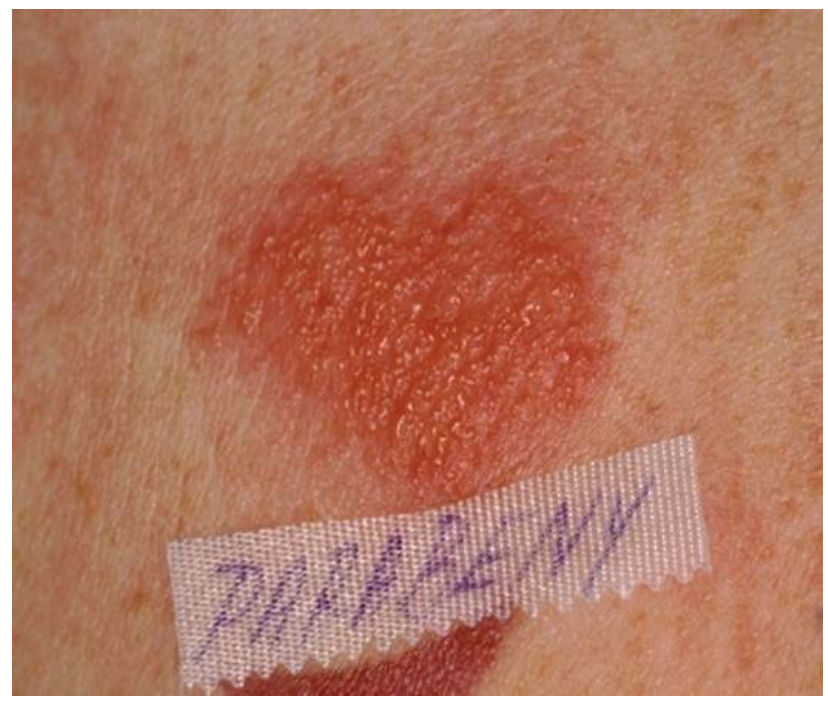

Figure 6: Positive patch test to parabens.

mercurial and a thiosalicylate. Although both components may cause allergic reactions, the proportion of ethylmercury in thimerosal generally acts as the allergic determinant [2]. Thimerosal hypersensitivity does not necessarily mean an allergy to mercury [3]. Reports of cross-reactions between thimerosal and other mercury components are infrequent $[2,3]$. Regarding the incidence of thimerosal allergies, there is a significant geographical variability. In the USA, the incidence of thimerosal allergies is as high as $15 \%$, while corresponding 
rates in Sweden and Denmark are about 3 to $4 \%[4,5]$. Such differences are due to variations in the availability and use of thimerosal-containing products in each country. The clinical relevance of thimerosal reactions is often controversial. A study conducted by the North American Contact Dermatitis Group (NACDG) found the prevalence and clinical relevance of thimerosal reactions to vary from $10.9 \%$ to $16.8 \%$, in the 4,087 individuals assessed [6]. The high prevalence of thimerosal reactions in population resulted in the American Contact Dermatitis Society identifying the substance to become "allergen of the year" in 2002. However, in the same year Belsito published an article declaring thimerosal to be the "non-allergen of the year", because of a lack of clinical relevance of such reactions and recommended its exclusion from the American skin test battery $[4,6]$. A group from Santa Casa of Belo Horizonte found that $14.7 \%$ of the 1,406 patients tested between 2003 and 2010 had positive thimerosal. Nevertheless, the report did not discuss the clinical relevance of the reactions [4]. Herbst, et al. confirmed allergies to thimerosal in a group of patients with periorbital dermatitis in $10.2 \%$ of the patients, yet with no comment regarding relevance [7]. Landeck, et al. conducted a retrospective analysis of data from patient's patch tested in Germany, Austria and Switzerland (IVDK Network) between January 2001 and December 2010 and found frequency of contact sensitization to thimerosal to be $5.8 \%$ [8]. Wijnmaalen, et al. performed a retrospective study in 90 patients with suspected allergy to ophtalmic medication, and they detected a contact allergy to thimerosal in $7 \%$ of the patients [9]. The frequency of contact sensitization to thimerosal in a cohort of 4,055 patients with chronic eczema tested between the years 2001 and 2016 in our department was found to be as high as $9.89 \%$. In this study, we found the frequency of contact sensitization to thimerosal in patients with periorbital dermatitis to be $9.7 \%$, but with very low clinical relevance (just one patient of 182). The use of thimerosal-containing ocular preparations may also lead to conjunctivitis, which may occur simultaneously with eyelid eczema, as well as corneal infiltration, epithelial punctate keratitis and corneal pseudodendrites [3]. Although thimerosal is present in hepatitis, tetanus and meningoencephalitis vaccines, significant adverse reactions to these substances are rare. Only $10 \%$ of thimerosal-sensitive patients report adverse reactions to these vaccines. However, these reactions are usually light to moderate, and are often limited to increased pain around the injection site [2,3,6,10-12].

Neomycin is an antibacterial that is used widely in topical creams, ointments, lotions, eye preparations and eardrops [13]. It is also commonly found in combined preparations with other antibacterials and corticosteroids. These prescription and non-prescription preparations are used to treat a variety of skin, eye and external ear disorders that have become infected and inflamed. It is also used as a preservative in some vaccines. Allergic contact dermatitis from neomycin was first described in 1952 [14]. Prystowsky, et al. determined the frequency of neomycin allergy in the general population to be $1.1 \%$ [15]. In 10 European centers, the sensitization rates varied from 1.1 do $3.8 \%$ [16]. American Contact Dermatitis Group revealed sensitization up to $11.6 \%$ [17]. Neomycin is therefore the fifth most common allergen in North America, largely because this antibiotic is widely available in various over-the-counter preparations in the USA. There may be a cross sensitivity with other antibacterials of aminoglycosid group which are chemically related, including framycetin, gentamicin, kanamycin, streptomycin, tobramycin, spectinomycin, paromomycin [13]. In the Czech Republic the general frequency of sensitization to neomycin sulfate was $1.2 \%$ in 2014 and $1.7 \%$ in 2016 among patch tested patients (data from the dermatoallergological section of the Czech Dermatovenerological Society). Herbst, et al. evidenced allergy to neomycin sulfate in a group of patients with periorbital dermatitis in 3.8\% [7]. Landeck, et al. conducted a retrospective analysis of data from patient's patch tested in Germany, Austria, and Switzerland (IVDK Network) between January 2001 and December 2010 and determined the frequency of contact sensitization to neomycin to be $5.9 \%$ [8]. Wijnmaalen, et al. conducted a retrospective study of 90 patients with suspected allergy to ophtalmic medication, and they detected a contact allergy to neomycin sulfate in $8 \%$ of the patients [9]. In our study, we have found very similar frequency of contact sensitization to neomycin sulfate (2.6\%) with quite significant clinical relevance (20 of 48 patients).

Benzalkonium chloride belongs to quarter ammonium bases. It is used as a preservative of cosmetics and extensively also in the pharmaceutical industry, e.g. for the preservation of eye drops and ointments as well as in solutions for preserving contact lenses. In eye drops the concentration ranges from 0.004 to $0.025 \%$ [18]. As a microbicidal agent, benzalkonium chloride is highly toxic in a time and dose dependent way [18]. But contact sensitization is supposed to be relatively rare $[2,19,20]$, for example Goosens reports it to be $0.02 \%$ [19], but among selected patients (having periorbital dermatitis) the frequency seems to be higher - Wijnmaalen, et al. detected a contact allergy to benzalkonium chloride in $4 \%$ of the patients [9], and Herbst, et al. found an allergy to this preservative in $1.9 \%$ of the patients [7]. Landeck, et al. conducted a retrospective analysis of data from patient's patch tested in Germany, Austria and Switzerland (IVDK Network) between January 2001 and December 2010 and affirmed a frequency of contact sensitization to benzalkonium chloride to be $1.6 \%$ [8]. The frequency of contact sensitization to benzalkonium chloride was determined to be $1.2 \%$ in a cohort of 2,400 patients with chronic eczema tested between the years 2001 and 2009 in our department [21] and 1.85\% 
among 4,055 patients with the same diagnosis tested from 2001 to 2016 also in our department. Also among our study patients with periorbital dermatitis the frequency of contact sensitization exceeded $1 \%(1.32 \%)$ with a clinical relevance being 3 out of 25 patients.

Parabens are derivatives of $\mathrm{p}$-hydroxy-benzoic acid. Methyl-, ethyl-, propyl-, butyl-, isobutyl-, and isopropyl ester are used both for the preservation of dermatological and cosmetic products. Sensitization in Czech Republic has decreased in recent years with the actual frequency of sensitization to be $0.8 \%$ in 2016 (data from the dermatoallergological section of Czech Dermatovenerological Society). Sensitization potential of parabens is relatively low, when considering long-term application. During 1999-2000 in 11 European countries, the frequency of sensitization was $0.5 \%$ [22], during 2004-2005 in Great Britain among 6,958 patients; sensitization was $0.8 \%$ [23]. Among our patients with periorbital dermatitis, sensitization was $0.6 \%$, however with no clinical relevance (none of the patients had used any ophtalmic product containing parabens).

\section{Conclusion}

The study has shown that sensitization to the excipients of ophthalmological topical preparations among patients with periorbital dermatitis is quite significant. The most frequently detected allergen was thimerosal, albeit with very low clinical relevance, followed by neomycin sulfate (conversely with markedly significant clinical relevance), benzalkonium chloride (relatively low clinical relevance), and parabens (no clinical relevance). Accordingly, regarding clinical relevance, the most important allergen in ophtalmic products is neomycin, followed by benzalkonium chloride.

Patch testing should be considered in every case of periorbital dermatitis, and not only with commercial test allergens but also with patient's actual ophthalmic preparations.

\section{Author Contributions}

Nečas and Dastychová performed patch tests, Nečas calculated sensitization frequency and wrote this article, Dastychová reviewed and checked the article.

\section{Statement of Competing Interests}

The authors state that there are no competing interests.

\section{Acknowledgements}

Technical assistance was provided by Nurse Pospíšilová and Lab Technician Licková with their preparation of allergens and application of patch tests.

\section{References}

1. Herbst RA, Maibach HI (1997) Allergic contact dermatitis from ophthalmics: update 1997. Contact Dermatitis 37: 252-253.

2. Rietschel R, Fowler JF (2008) Fisher's Contact Dermatitis.
(6 $6^{\text {th }}$ edn), Hamilton, Bc Deckerinc, Canada.

3. van't Veen AJ, van Joost T (1994) Sensitization to thimerosal (merthiolate) is still present today. Contact Dermatitis 31: 293-298.

4. Rodrigues DF, Neves DR, Pinto JM, Alves MF, Fulgêncio AC (2012) Results of patchtestsfrom santa casa de belo horizonte dermatology clinic, belo horizonte, brazil, from 2003 to 2010. An Bras Dermatol 87: 800-803.

5. Moriearty P, Pereira C, Guimarães NA (1978) Contact dermatitis in salvador, Brazil. Contact Dermatitis 4: 185-189.

6. Belsito DV (2002) Thimerosal: contact (non)allergen of the year. Am J Contact Dermat 13: 1-2.

7. Herbst RA, Uter W, Pirker C, Geier J, Frosch PJ (2004) Allergic and non-allergic periorbital dermatitis: patch test results of the information network of the Departments of Dermatology during a 5-year period. Contact Dermatitis 51: 13-19.

8. Landeck L, John SM, Geier J (2014) Topical ophthalmic agents as allergens in periorbital dermatitis. $\mathrm{Br} \mathrm{J}$ Ophthalmol 98: 259-262.

9. Wijnmaalen $A L$, van Zuuren EJ, de Keizer RJ, Jager MJ (2009) Cutaneous allergy testing in patients suspected of an allergic reaction to eye medication. Ophthalmic Res 41: 225-229.

10. Möller H (1994) All these positive tests to thimerosal. Contact Dermatitis 31: 209-213.

11. Stingeni L, Lapomarda V, Lisi P (1996) What risk of piroxicam photodermatitis in thimerosal-positive patients? Contact Dermatitis 34: 60-61.

12. Breithaupt A, Jacob SE (2008) Thimerosal and the relevance of patch-test reactions in children. Dermatitis 19: 275-277.

13. Sasseville D (2010) Neomycin. Dermatitis 21: 3-7.

14. Baer R, Ludwig JS (1952) Allergic eczematous sensitization to neomycin. Ann Allergy 10: 136-137.

15. Prystowsky SD, Nonomura JH, Smith RW, Allen AM (1979) Allergic hypersensitivity to neomycin. Relationship between patch test reactions and 'use' tests. Arch Dermatol 115: 713-715.

16. Uter W, Rämsch C, Aberer W, Ayala F, Balato A, et al. (2009) The European baseline series in 10 European Countries, 2005/2006--results of the European Surveillance System on Contact Allergies (ESSCA). Contact Dermatitis 61: 31-38.

17. Zug KA, Warshaw EM, Fowler JF Jr, Maibach HI, Belsito DL, et al. (2009) Patch-Test Results Of The North American Contact Dermatitis Group 2005-2006. Dermatitis 20: 149-160.

18. Baudouin C, Labbé A, Liang H, Pauly A, Brignole-Baudouin $F$ (2010) Preservatives in eyedrops: the good, the bad and the ugly. Prog Retin Eye Res 29: 312-334.

19. Goossens A, Claes L, Drieghe J, Put E (1997) Antimicrobials: preservatives, antiseptics and disinfectants. Contact Dermatitis 39: 133-134.

20. Pecquet C, Bayrou O, Leynadier F (2002) Prevalence of positive patch tests with preservatives. Contact Dermatitis 58: 73.

21. Dastychová E, Necas M (2010) Kontaktní precitlivelost na pomocné látky prípravku kosmetických a farmaceutických u ekzematiku. Ces-Slov Derm 85: 78-90.

22. Wilkinson JD, Shaw S, Andersen KE, Brandao FM, Bruynzeel DP, et al. (2002) Monitoring levels of preservative sensitivity in Europe. A 10-year overview (1991-2000). Contact Dermatitis 46: 207-210.

23. Jong CT, Statham BN, Green CM, King CM, Gawkrodger DJ, et al. (2007) Contact sensitivity to preservatives in the uk, 2004-2005: results of multicentre study. Contact Dermatitis 57 : 165-168. 